\title{
Método para Promover el Aprendizaje Colaborativo en Ingeniería de Software
}

\author{
Héctor C. Cornide-Reyes ${ }^{(1)}$ y Rodolfo H. Villarroel(2) \\ (1) Departamento de Ingeniería Informática y Ciencias de la Computación, Universidad de Atacama, Avenida Copayapu \\ 485, Copiapó, Chile. (e-mail: hector.cornide@uda.cl) \\ (2) Escuela Ingeniería Informática, Pontificia Universidad Católica de Valparaíso, Avenida Brasil 2241, Valparaíso, Chile. \\ (e-mail: rodolfo.villarroel@pucv.cl)
}

Recibido Sep. 11, 2018; Aceptado Dic. 4, 2018; Versión final Ene. 24, 2019, Publicado Ago. 2019

\begin{abstract}
Resumen
Este artículo presenta la creación de un ambiente de aprendizaje colaborativo mediante la integración del método BYOS (Build your own Scrum, Crea tu propio Scrum) al modelo pedagógico Flipped Classroom en Ingeniería de Software. Esta experiencia aprovecha los beneficios del modelo Flipped Classroom con la incorporación de un método que estimule el aprendizaje colaborativo de los estudiantes en el aula. El objetivo de esta investigación es propiciar el aprendizaje colaborativo mediante la integración del método BYOS al modelo Flipped Classroom en la enseñanza de los conceptos de Scrum. Los resultados obtenidos son alentadores debido a que el método aplicado facilitó la comprensión de los contenidos y la percepción de los alumnos fue positiva respecto al aprendizaje colaborativo. Los resultados también señalan que el tiempo debe ser bien controlado para lograr los objetivos en este tipo de innovaciones pedagógicas realizadas en el aula.
\end{abstract}

Palabras clave: clase invertida; aprendizaje activo; aprendizaje colaborativo; ingeniería de software

\section{Method to Promote Collaborative Learning in Software Engineering}

\begin{abstract}
This article presents the creation of a collaborative learning environment through the integration of the BYOS method (Build your own Scrum) to the Flipped Classroom pedagogical model in Software Engineering. This experience takes advantage of the benefits of the Flipped Classroom model with the incorporation of a method that stimulates the collaborative learning of the students in the classroom. The objective of this research is to promote collaborative learning by integrating the BYOS method to the Flipped Classroom model in the teaching of Scrum concepts. The results obtained are encouraging because the applied method facilitated the understanding of the contents and the perception of the students was positive regarding collaborative learning. The results also indicate that the time should be well controlled to achieve the objectives in this kind of pedagogical innovations in the classroom.
\end{abstract}

Keywords: flipped classroom; active learning; collaborative learning; software engineering 


\section{INTRODUCCIÓN}

A pesar de que en la web existen muchos recursos disponibles para realizar innovaciones en el aula, en la práctica, el proceso de enseñanza-aprendizaje continúa desarrollándose mayormente bajo un esquema tradicional, donde el profesor es el centro y los alumnos son entes pasivos en su aprendizaje. Este tipo de modalidad está cambiando bajo la perspectiva constructivista en el desarrollo del aprendizaje y a la incorporación de métodos activos en la enseñanza de la Ingeniería de Software (López et al., 2016). En la literatura existen muchas evidencias en las cuales se intentan crear ambientes de trabajo colaborativo en el aula, para dejar a un lado el ambiente individualista que se presentan en la mayoría de los cursos de Ingeniería de Software. Un buen ejemplo de lo anterior, es el trabajo de Shim et al., (2009) donde sugieren que la enseñanza de la Ingeniería de Software no ha de hacerse orientada a la individualidad del sujeto, sino que necesita que el alumno desarrolle habilidades humanas para la comunicación, interacción y colaboración entre miembros del equipo, siendo esto esencial para el éxito de los proyectos de software y derivando en la aplicación de una metodología colaborativa para la enseñanza.

El presente artículo, trata sobre la aplicación del modelo Flipped Classroom, en adelante FC, (Bergmann y Sams,2012; Chen et al., 2014) más conocido como "Clase Invertida" o "Aula Invertida" junto al método colaborativo BYOS (Build Your Own Scrum, Crea tu propio Scrum), en el curso de Ingeniería de Software de las carreras de Ingeniería Civil Informática e Ingeniería de Ejecución en Informática de la Pontificia Universidad Católica de Valparaíso (PUCV). Se ha seleccionado el modelo FC para este curso, ya que favorece el logro de los aprendizajes esperados, promoviendo que las categorías de orden inferior (recordar y comprender) sean alcanzadas por el estudiante fuera del aula, mientras que en el aula se focaliza la atención en el logro de las categorías de orden superior (aplicar, analizar, evaluar y crear) de la taxonomía de Anderson et al. (2001), de tal forma que el docente pueda estar presente en el momento más importante y relevante del proceso de aprendizaje de sus estudiantes para orientarlos, apoyarlos y retroalimentarlos oportunamente (Johnson y Renner, 2012). En resumen, este modelo permite no solo la adquisición de conocimientos y habilidades, sino también el desarrollo de las habilidades necesarias para manipularlas y aplicarlas en contextos nuevos. Por otra parte, este modelo promueve el aprendizaje activo y la autonomía de los estudiantes, mientras que el docente se convierte en un guía y facilitador en lugar de un mero transmisor de conocimiento. Además, fomenta la interacción social y el aprendizaje colaborativo entre los estudiantes dándoles especial relevancia a estos aspectos. En trabajos anteriores (Griffiths et al., 2016; Villarroel et al., 2017), se han incorporado algunas herramientas TIC (Plickers y Kahoot!) para estimular el trabajo colaborativo y como complemento al modelo FC para las evaluaciones formativas. Si bien, estas herramientas mostraron un alto nivel de aceptación entre los alumnos, la estimulación del trabajo colaborativo no siempre fue lograda. Por tal razón, se investigaron algunas alternativas que pudieran ayudar a crear ambientes colaborativos de trabajo en el aula.

El uso de tecnología con lápiz y papel, proporciona una forma simple y de muy bajo costo, de construir y propiciar ambientes enriquecidos por la constante interacción de quienes participan y por el hecho de visibilizar la construcción del aprendizaje. Las ventajas en el uso de este tipo de tecnologías son: 1) Promueve la interacción rápida: se trabaja en tiempo real; 2) Es económico. Solo se requieren papeles, tijeras y lápices; 3) Aumenta la creatividad: Ios estudiantes visualizan sus ideas en el prototipo; 4) Promueve el trabajo en equipo: se necesita formar grupos de trabajo; 5) Permite aprendizajes multidisciplinarios al promover la interacción entre los distintos miembros del grupo. Por lo anterior, se planificó aplicar el modelo FC a una clase sobre "Conceptos de Scrum" e integrar el método BYOS para lograr en los alumnos un pensamiento más reflexivo sobre SCRUM. Este trabajo es un nuevo intento por ganar experiencia académica en metodologías activas y para evaluar la efectividad del método BYOS en el modelo FC. Este artículo está organizado como sigue: se incluye una descripción del modelo FC y del método BYOS, luego la metodología de esta experiencia, la discusión de los resultados y finalmente las conclusiones.

\section{MODELO FLIPPED CLASSROOM Y MÉTODO BYOS}

El modelo FC está recibiendo cada vez mayor atención en círculos dedicados a la educación y en prensa especializada (Johnson y Renner, 2012) gracias a los beneficios y efectividad que está comenzando a demostrar (Bennett et al., 2012; Talbert, 2012; Rivero-Guerra, 2018). Existen varias definiciones en la literatura sobre FC, invertir la clase significa que los acontecimientos que tradicionalmente han tenido lugar en el aula ahora se llevarán a cabo fuera del aula, y viceversa (Lage et al., 2000). Esta definición establece que este modelo representa una simple reorganización de las actividades dentro y fuera del aula, sin embargo su aplicación práctica ha demostrado que es más que eso y que puede tener múltiples variaciones (Foertsch et al. ,2002; Lage et al., 2000; López Rodríguez et al., 2016; Prieto et al., 2016; Sáez y Ros, 2014; Tourón y Santiago, 2015). Precisando la definición, invertir el aula significa que los estudiantes adquieren el primer contacto con un nuevo material fuera del aula, por lo general a través de lecturas o lecciones en vídeo, y luego el tiempo en aula se usa para realizar el trabajo más difícil de asimilación del conocimiento a través de 
estrategias tales como la resolución de problemas, desarrollo de proyectos, estudio de casos, discusión, debate, etc. (Brame, 2013).

FC es un modelo didáctico-pedagógico que incluye actividades de aprendizaje significativo, personalizado y colaborativo que se realizan en el aula, e instrucción individual directa realizada en computador fuera del aula. Una característica importante de este modelo es el cambio en los roles profesor-estudiante con respecto al modelo tradicional. Es decir, el profesor asume un rol de guía, facilitador y colaborador durante el proceso de aprendizaje de los estudiantes y deja de ser la fuente única de conocimiento. Los estudiantes por su parte asumen un rol activo y protagónico en el proceso, ya que no solo son responsables de la adquisición y asimilación de los contenidos de manera previa a la clase, sino que deben participar en las diversas actividades prácticas realizadas en el aula en colaboración con sus pares. Otro atributo destacable respecto al modelo tradicional es que en FC se pasa de una mera cobertura del contenido al dominio de los mismos por parte de los estudiantes. Finalmente, se puede destacar que existen evidencias en el área de las ciencias, tecnología, ingeniería y matemáticas en el ámbito universitario que prueban las ganancias significativas de aprendizaje que el modelo FC puede producir en comparación a una estructura de clase tradicional (Berrett, 2012; Deslauriers et al., 2011; Moravec et al., 2010). Uno de los desafíos que todo docente debe enfrentar es lograr que los alumnos interioricen conceptos teóricos relacionados con la materia. La disciplina de la Ingeniería en Software, no es ajena a esta dificultad, más aún cuando gran parte del contenido trata sobre técnicas, modelos y metodologías.

El método BYOS, fue creado el año 2011 por Adam Weisbart, quien es un reconocido Coach internacional de Scrum. Éste método consiste en armar una representación de Scrum utilizando unos ítems representados en tarjetas donde están los componente del marco de trabajo ágil. Todo este material es gratuito y se puede descargar directamente en la página del autor. Los materiales necesarios para cada grupo que realice esta actividad son: 01 Set de tarjetas BYOS, 01 pliego de cartulina, Post-it de colores, Cinta adhesiva y Plumones. El objetivo de BYOS es lograr que cada grupo se auto-organice y logren conceptualizar a través de un esquema el framework de Scrum. Para lo anterior, es necesario formar grupos entre 5 y 6 personas, quienes deben construir juntos un concepto y representarlo de una manera que sea fácil de comprender por otros alumnos. Una de las gracias de este método, es que propone un trabajo iterativo en el desarrollo de la experiencia con instancias de retroalimentación y mejora de lo realizado. Fueron precisamente estas características especiales que llamaron nuestra atención y gatillaron la decisión de utilizarla en conjunto al modelo FC.

\section{METODOLOGÍA}

Para poder desarrollar esta experiencia de FC junto a BYOS, se desarrollaron las siguientes etapas: 1) Definir objetivo de la experiencia; 2) Descripción de la Asignatura y alumnos participantes; 3) Pre-clase; 4) Clase Práctica Presencial (Evaluación formativa y Método BYOS); 5) Post-clase; 6) Aplicación de la encuesta de evaluación; 7) Análisis y discusión de resultados.

\section{Definir el objetivo de la experiencia}

El objetivo principal es: Propiciar el aprendizaje colaborativo mediante la integración del método BYOS al modelo FC en la enseñanza de los conceptos de Scrum en la asignatura de Ingeniería de Software para las carreras de Ingeniería Civil Informática e Ingeniería de Ejecución en Informática de la PUCV. Como objetivo secundario, se propone: Identificar las bondades y las barreras existentes al momento de incorporar este tipo de métodos activos de enseñanza-aprendizaje al modelo FC. Las habilidades que serán observadas durante la realización de esta experiencia serán: Colaboración, Auto-aprendizaje, Compromiso y motivación. Dentro de los beneficios que se esperan conseguir, se encuentran los siguientes: 1) La percepción de los alumnos sobre los beneficios que tiene FC en su proceso formativo; 2) El desarrollo de aprendizaje autónomo y colaborativo; 3) El ambiente de aprendizaje colaborativo utilizando recursos de bajo costo.

\section{Descripción de la Asignatura y alumnos participantes}

Esta experiencia, fue aplicada a dos grupos distintos: el primero fue de 52 alumnos pertenecientes a la carrera Ingeniería Civil Informática y, el segundo grupo, de 25 alumnos pertenecientes a la carrera de Ingeniería de Ejecución en Informática. Ambos grupos, cursaban la asignatura de Ingeniería de Software durante el primer semestre del año 2018. Ingeniería de Software es una asignatura de carácter obligatorio, correspondiente al área de Ingeniería Aplicada. Ingeniería de Software proporciona al estudiante una visión integrada del desarrollo del software, como una rama de la ingeniería. El estudiante debe comprender y aplicar técnicas de ingeniería en todas las etapas del ciclo de vida del software. Predominantemente, el curso de Ingeniería de Software se ha impartido utilizando una metodología tradicional centrada en la clase magistral. La metodología tradicional está orientada principalmente a que el estudiante reciba de manera efectiva un conjunto de 
conocimientos, pero no enfatiza los aspectos procedimentales y actitudinales necesarios en la formación de cualquier graduado en el ámbito de la ingeniería. De allí que la búsqueda, implementación y evaluación de nuevas metodologías activas centradas en el aprendizaje sea una tarea obligatoria, necesaria y fundamental para esta asignatura.

El modelo FC, mayormente se ha utilizado cuando se han detectado dificultades en la asimilación de contenidos. En esta experiencia de investigación, se utilizará con el objeto de propiciar un ambiente de trabajo colaborativo entre los alumnos. Para tal efecto y, dentro del programa de la asignatura de Ingeniería en Software, se ha seleccionado el contenido sobre "Conceptos de Scrum" como tema para desarrollar la experiencia de aprendizaje.

\section{Pre-Clase}

A pesar de que los alumnos de las carreras Ingeniería Civil Informática e Ingeniería de Ejecución en Informática, han tenido en su mayoría experiencias previas en FC, se realizó una pequeña charla a los alumnos de los cursos sobre el modelo, las actividades a realizar y los nuevos roles que desarrollarían tanto el profesor como los alumnos. Un aspecto clave es la determinación y preparación del material a entregar a los alumnos. Esta actividad suele consumir mucho tiempo de preparación, porque debe cumplir una serie de objetivos, siendo el más importante lograr comprometer a los alumnos con su propio aprendizaje. Por lo anterior, se determinó trabajar con tres tipos de materiales: 1) Video Introductorio: El objetivo es presentar al estudiante una visión general del contenido a tratar. La duración debe estar entre los 7 y 10 minutos. Debe estimular al alumno con la materia a estudiar. Este video es subido a la plataforma Youtube; 2) Contenido teórico: A través de diapositivas, a los alumnos se les entregó la teoría que debían estudiar. Este formato se utiliza para facilitar la organización del contenido, lectura y comprensión de los conceptos; 3) Artículo Complementario: Este material, tiene por objetivo proporcionar al alumno una visión aplicada de los contenidos plasmados en las diapositivas y, lo ideal, es que sea un artículo de alguna revista científica reconocida en el área.

En el modelo FC, la pre-clase tiene una duración mínima de una semana, y se inicia con la habilitación del material en el Navegador Académico, que es una plataforma LMS (Learning Management System, Sistema de Gestión de Aprendizaje) institucional, dispuesta para que los alumnos accedan, entre otras cosas, al material de cada asignatura que tienen inscrita. Durante la pre-clase, los alumnos estudian de forma autónoma utilizando el material entregado. Lo ideal es que el material entregado logre cautivar y motivar al alumno para que pueda explorar por su cuenta y busque más conocimiento en el área de estudio. También se entrega la posibilidad de hacer consultas al profesor en el caso de que existan dudas en la comprensión del material, aunque se les insta a que puedan resolver por sí solos este tipo de dificultades.

\section{Clase Práctica Presencial}

Durante la clase práctica, se desarrollaron dos actividades orientadas al trabajo colaborativo. Como primera actividad, se realizó una evaluación formativa que tenía por objetivo verificar el nivel de conocimientos previos de los alumnos y, por ende, controlar si habían seguido las instrucciones, es decir, ver el video introductorio, estudiar las diapositivas y leer el artículo complementario. Esta evaluación se realizó de forma grupal y consistió en dos preguntas cortas que invitaban al debate en el grupo. Para lo anterior, se definió un tiempo de 10 minutos para discusión/conversación de las preguntas y 5 minutos para escribir las respuestas. La segunda parte de la clase práctica, consistió en el desarrollo de actividades según lo propuesto por el método BYOS, ajustando los tiempos de las iteraciones debido a las limitaciones existentes por los horarios de las clases. La figura 1 muestra las fases del método y los tiempos ajustados para esta experiencia. Cabe destacar que la principal amenaza para el éxito de esta experiencia, fue lo ajustado del tiempo para cada una de las fases establecidas por el método. Sin embargo, la buena disposición y alto sentido de colaboración de los alumnos permitieron desarrollar cada una de las fases dentro de los tiempos establecidos.

La figura 2(a), muestra a un grupo de alumnos trabajando colaborativamente durante la primera fase del método. Durante esta fase, se observaron distintas estrategias para desarrollar el trabajo, las que a juicio de los observadores, fueron influenciados por el nivel de conocimiento que tenían sobre la materia. Aquellos alumnos que estudiaron de forma adecuada, alcanzaron mejores niveles de cumplimiento durante esta fase, a diferencia de aquellos grupos que no tenían un buen dominio de la materia o estudiaron muy poco antes de la clase. Esto último se hacía evidente al observar a algunos alumnos revisando el material enviado o buscando en internet algo que les pudiera ayudar a conseguir lo requerido. La segunda fase estuvo caracterizada por el trabajo colaborativo entre los distintos grupos. Esta retroalimentación fue muy provechosa para todos los alumnos porque debían intentar explicar a otras personas el trabajo realizado, lo que les permitió detectar algunos errores conceptuales, así como también, problemas de representación de la idea. Esta actividad también entregó la posibilidad de enfrentar de mejor forma la tercera fase, debido a que la 
confianza en el conocimiento creado aumentó respecto a la primera fase. Se pudo observar que la retroalimentación ayudó a los equipos a mejorar el trabajo realizado al copiar ideas de representación observadas en otros equipos.

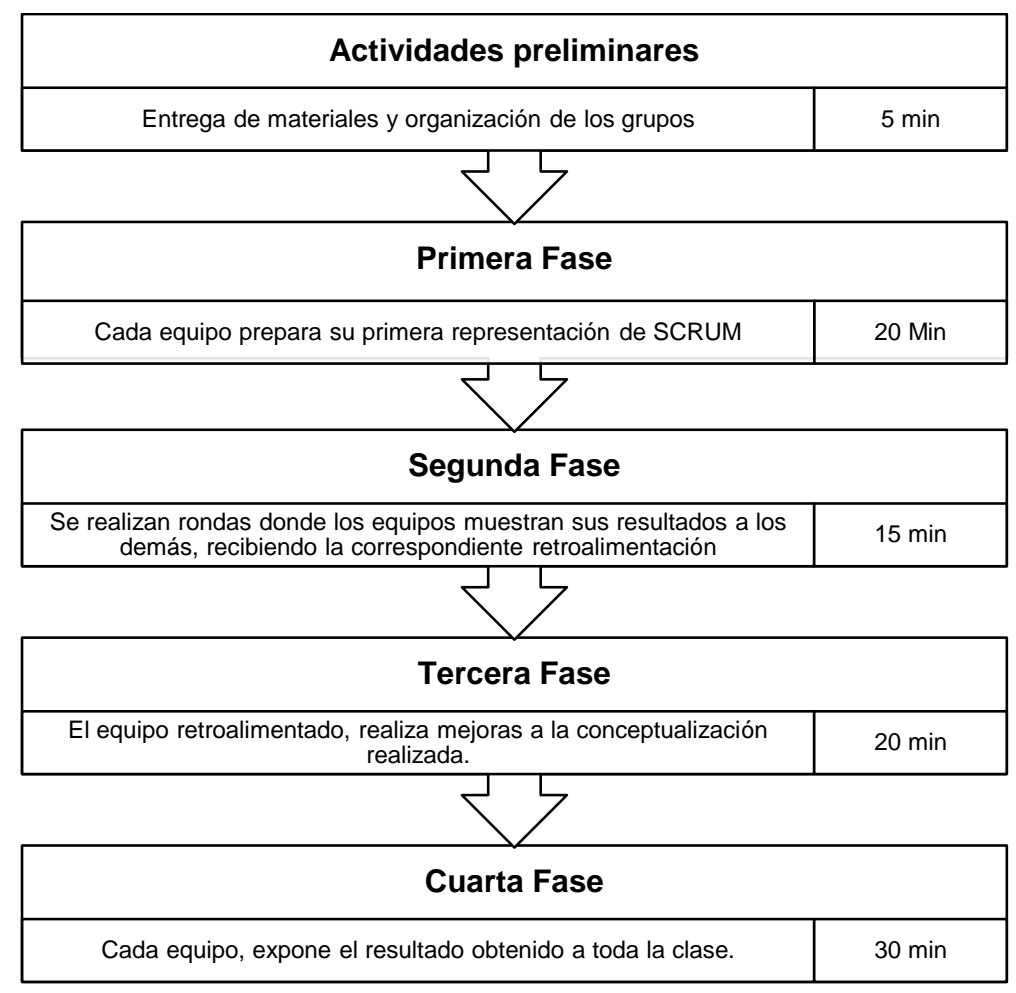

Fig. 1: Método BYOS aplicado

La figura 2(b) muestra a los alumnos interactuando durante esta fase. La figura 2(c) muestra a alumnos representantes de un equipo mostrando y explicando con breves palabras el resultado obtenido. Cabe destacar que no existe una única solución al problema planteado en el método BYOS, y obtuvimos soluciones creativas y únicas de representación. Lo anterior, al ser observado también por los alumnos, generó un mayor nivel de confianza en el trabajo que estaban realizando. Resultó llamativo observar que cuando los alumnos se enteran de que las actividades no tienen una única solución, trabajan menos presionados porque se concentran en lo que están haciendo y no en lo que el profesor quiere que hagan.

\section{Post-Clase}

La actividad de post-clase, se desarrolló en forma grupal y consistió en la realización de un ensayo con estructura definida (Portada - Introducción - Desarrollo del Tema - Conclusiones-Bibliografía) y que buscó profundizar en los conocimientos sobre métodos ágiles. Para la realización de esta actividad se destinaron 10 días, con el objeto de no tener impedimentos en investigar adecuadamente y entregar el informe en la calidad y oportunidad requerida. En total, se conformaron 12 grupos para los alumnos de Ingeniería Civil Informática y 6 grupos para Ingeniería de ejecución en Informática. Los temas asignados fueron: ¿Cómo se gestiona el product backlog?; ¿Cómo hacer una retrospectiva?; ¿Cómo se realiza la estimación de esfuerzo en Scrum?, ¿Qué son y cómo se gestionan las historias de usuario?; ¿Qué habilidades se necesitan para un buen Scrum Master?; ¿Qué habilidades se necesitan para un buen Product Owner?; ¿Scrum v/s ITIL o Scrum+ITIL?; Análisis comparativo de Scrum y XP; Casos de éxito de Scrum en Chile, ¿Qué es AgileRE?, ¿Cómo se realiza la planificación de un sprint?, ¿Cuáles son las claves para que las reuniones diarias sean productivas?

Para la evaluación de los ensayos, se ponderó de forma individual cada parte del documento utilizando la siguiente rúbrica: Nota 1; No cumple lo requerido o No realizado; Nota 3: Medianamente cumple lo requerido; Nota 5: Cumple lo requerido con modificaciones y Nota 7: Cumple satisfactoriamente lo requerido. La nota final de cada grupo, se calculó considerando la nota de cada parte del documento y el peso definido. Los pesos definidos para cada parte del documento son: Formato y presentación (15\%), Desarrollo del tema $(70 \%)$, Conclusiones (10\%) y Bibliografía (5\%). Los trabajos finales, se distribuyeron entre todos los alumnos del curso. 


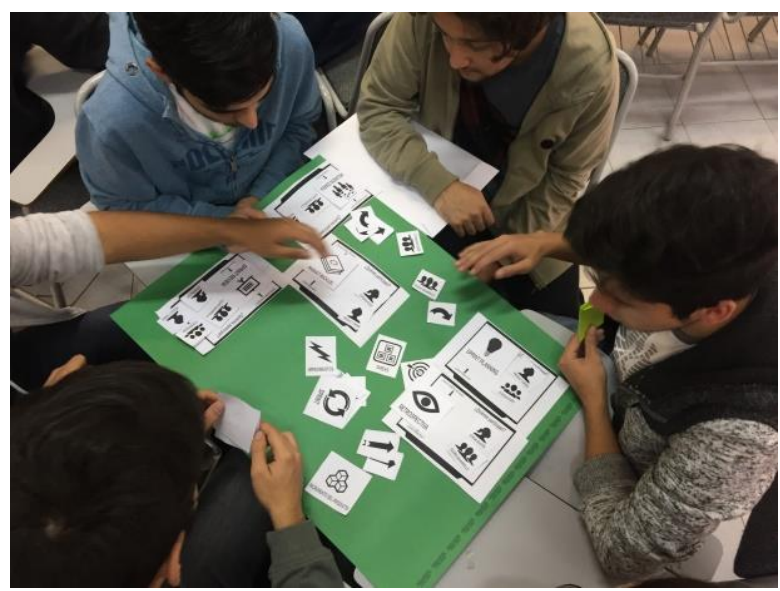

(a)

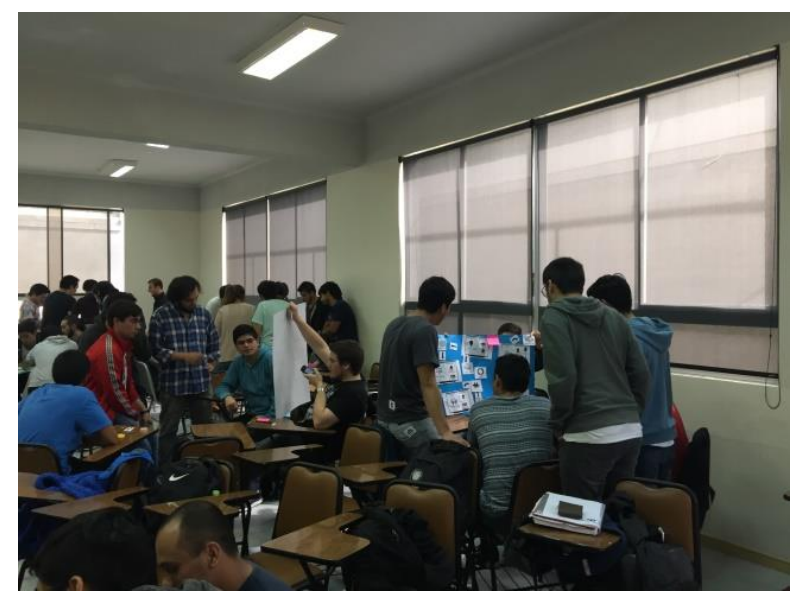

(b)

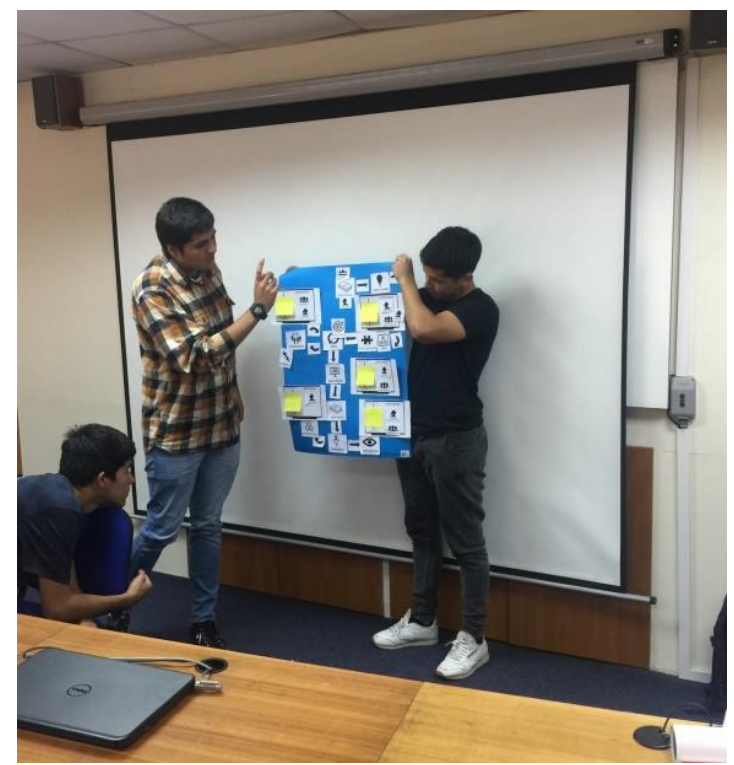

(c)

Fig. 2: Alumnos desarrollando las fases del Método BYOS.

Aplicación de la encuesta de evaluación

Una vez corregidos, evaluados e informados los trabajos de la post-clase se envió, a través del Navegador Académico, la encuesta de evaluación planificada para esta actividad, cuyos resultados serán presentados en la siguiente sección. La encuesta fue creada mediante Google Forms y consideró las siguientes preguntas: 1) Prefiero tener la clase tradicional del profesor en vez de realizar trabajos activos y grupales en clases como los que se llevaron a cabo con metodología Flipped Classroom (Clase invertida). 2) ¿Crees que el material entregado (ppt, video y paper) permitió un buen aprendizaje de los contenidos. 3) ¿Tuviste tiempo suficiente para estudiar el material enviado antes de la clase? 4) A partir del material inicial enviado, ¿buscó información adicional en la web que le permitiera comprender de mejor forma el tema? 5) ¿Cómo evalúas la actividad práctica grupal realizada en el aula? 6) La actividad práctica grupal realizada en el aula, ¿Mejoró tu nivel de conocimiento respecto a Scrum? 7) Por favor, escribe aquí cualquier comentario y/o sugerencia que nos permita mejorar este tipo de metodologías de aprendizaje.

\section{DISCUSIÓN DE RESULTADOS}

En esta experiencia de aprendizaje en la asignatura de Ingeniería de Software, se ha elegido el modelo FC en conjunto al método colaborativo BYOS, teniendo en consideración las necesidades y desafíos de aprendizaje que plantean en la actualidad los estudiantes universitarios (Núñez et al., 2014; Arbizy y Lobato, 2003).

Para analizar la evaluación de los profesores, se revisaron las anotaciones en papel realizadas durante la experiencia, las que fueron analizadas y discutidas luego de finalizar las actividades. El equipo de observación estaba constituido por dos profesores de Ingeniería de Software junto a los dos ayudantes de la asignatura. Para analizar la evaluación de los alumnos, se revisaron las opiniones y respuestas que ellos emitieron en la encuesta de satisfacción aplicada al final de la postclase. 


\section{Respecto a los objetivos y resultados esperados}

Los objetivos propuestos en esta investigación se lograron de forma satisfactoria, debido a que se logró crear un ambiente que propició el aprendizaje colaborativo de los estudiantes. En los buenos resultados mostrados en la integración del método BYOS a la FC, influyó considerablemente el alto nivel de compromiso de los estudiantes con la actividad y con su autoaprendizaje, lo cual está en correspondencia con los resultados de otros investigadores (Rivero-Guerra, 2018; Estrada et al., 2016; Griffiths et al., 2016; Villarroel et al., 2017; Hernández et al., 2014).

Se han obtenido aprendizajes y conclusiones importantes luego de realizar la experiencia, lo que nos ayudará a desarrollar en el futuro nuevas experiencias innovadoras en esta materia.

\section{Resultados por parte de los profesores}

Por parte de los profesores la valoración sobre el modelo de FC es muy positiva, así como también, la utilidad del método BYOS como instancia para propiciar el aprendizaje colaborativo en los alumnos. Por lo observado en el desarrollo de las actividades y, de acuerdo a los resultados en la evaluación formativa, los alumnos mostraron un buen nivel de conocimiento en la materia. Algo que llamó la atención en la experiencia, fue la existencia de algunas diferencias en las habilidades genéricas de los alumnos de Ingeniería Civil en Informática respecto a los alumnos de Ingeniería de Ejecución en Informática. Por ejemplo, se observa que la habilidad de "Autoaprendizaje" mostró un mejor nivel de logro en estudiantes de Ingeniería Civil en Informática en comparación a los alumnos de Ingeniería de Ejecución en Informática. Algo similar ocurrió al observar otras habilidades como "Comunicación Efectiva" y "Compromiso con la actividad". Los niveles observados de "Colaboración" fueron muy similares en ambos grupos de alumnos. En general, los profesores reconocen que los alumnos adoptan de manera muy positiva este tipo de innovaciones pedagógicas, mostrándose siempre muy entusiastas y motivados con las actividades. Sin duda, FC logra mejoras significativas en la interacción alumno-profesor y alumno-alumno. Este modelo requiere que el alumno sea un agente activo en su aprendizaje, por lo que se propician interacciones entre alumno y profesor mucho más provechosos y enriquecedores para ambos. Algo similar ocurre en las interacciones alumno-alumno, debido a que este modelo genera un ambiente para el desarrollo de aprendizaje colaborativo.

El uso del método BYOS resultó ser muy positivo, porque propició un espacio donde los alumnos pudieron generar conversaciones poderosas con su equipo. En las iteraciones realizadas, fue posible observar cómo los alumnos ayudaban a corregir algunos errores de otros equipos, así como también, darse cuenta de algunos errores que ellos habían cometido en sus respectivos equipos. Al revisar las respuestas de la evaluación formativa aplicada, los profesores lograron deducir que los alumnos no tan sólo habían seguido las instrucciones, sino que también, el trabajo autónomo les permitió iniciar la clase con una mejor comprensión de los contenidos. La observación en el aula y, los resultados obtenidos por los equipos, permitieron evaluar cualitativamente las habilidades de compromiso con la actividad y de autoaprendizaje. Estas habilidades, mostraron un buen nivel de logro en la gran mayoría de los alumnos, en comparación a las experiencias anteriores en la entrega de los mismos contenidos bajo el método tradicional. En el futuro, estamos planificando la incorporación de técnicas de análisis multimodal del aprendizaje para obtener datos más cuantitativos que cualitativos.

\section{Resultados por parte de los alumnos}

La encuesta fue aplicada a ambos grupos (75 alumnos) y fue respondida por $2 / 3$ de ellos. Al revisar los resultados y opiniones entregados por los alumnos, es posible confirmar gran parte de lo observado por los profesores durante la experiencia. En lo que refiere al nivel de aceptación del modelo FC por parte de los alumnos, un $82 \%$ de ellos prefiere este tipo de metodología activa en desmedro de la metodología tradicional.

Frente a la pregunta: ¿Cómo evalúas la actividad práctica realizada en el aula?, donde se solicitaba colocar una nota (escala 1 a 10), los alumnos valoran muy positivamente la actividad práctica y un $85 \%$ de los alumnos respondió con una nota sobre 7 . Esta cifra, confirma lo observado por los profesores, en el sentido de que la actividad resultó muy atractiva para los alumnos, percibiendo de muy buena forma el conocimiento adquirido durante esta experiencia. Frente a la pregunta: ¿Crees que el material entregado (presentación de diapositivas, video y paper) permitió un buen aprendizaje de los contenidos? Un 75,5\% (31,1\% Totalmente de acuerdo $+44,4 \%$ De acuerdo) de los alumnos evalúa en forma muy positivamente el material entregado. Respecto a la pregunta: La actividad práctica grupal realizada en el aula, ¿Mejoró tu nivel de conocimiento respecto a SCRUM? Un $86,7 \%$ de los alumnos le otorga un valor positivo al método BYOS en la mejora del conocimiento que tenían hasta antes de la actividad práctica 
A continuación, se muestran algunos extractos de comentarios realizados por los alumnos en la encuesta aplicada: "Me gustó mucho la actividad ya que al ser algo novedoso dentro de lo que estamos acostumbrados, nos hizo ser más participativos."; "En lo personal, encuentro que estas prácticas de aprendizaje son fantásticas para la fácil comprensión de los temas ya que el contenido se ve de una forma dinámica y didáctica."; "No perder estas actividades debido a que permiten aprender de una manera distinta a la tradicional."; "El traer a personas externas, como fue el caso de la clase Invertida , ciertamente ayudó a estimular la parte creativa y mejorar el dinamismo (creo yo) al ver personas, ideas y visiones nuevas sobre la ingeniería de software, que al final es de lo que se va a tratar en la práctica."; "Designar más tiempo para la actividad y no solo la hora de catedra."; "Me parece buena la iniciativa de poder ir conociendo de manera más didáctica en cómo se pueden ir aplicando las diferentes técnicas para organizar un equipo de trabajo y, sobretodo, conocer técnicas para aplicarlas en el desarrollo del software en ese contexto la metodología Scrum que actualmente muchas empresas las están utilizando."; "Ojala siguiera así."; "Encontré que fue bastante buena, hubiera sido bueno haber visto un ejemplo que hubiera armado el profesor."; "el tiempo durante la clase para poder realizar el trabajo es muy corto a mi parecer, en mi grupo discutimos todo, sin embargo el tiempo nos hizo tomar decisiones prematuras para la organización de las cosas."

\section{CONCLUSIONES}

Con nuestra experiencia, fue posible reforzar la idea de que el modelo FC es muy útil cuando los contenidos de cierta asignatura presentan dificultades de comprensión y entendimiento para los alumnos. El método BYOS tuvo una mejor recepción de la esperada. La innovación pedagógica no siempre necesita de buenas TIC para tener una buena recepción por parte de los alumnos. Existen en la web diversos métodos creados por expertos que no deberían ser descartados por sólo utilizar materiales simples como cartulinas, post-it, cintas adhesivas y plumones. Es necesario analizar las restricciones de tiempo que existen en las carreras con bloques fijos (90 minutos) para las clases, debido a que aparece como una limitante importante a la hora de realizar innovaciones en el aula. Este elemento fue observado por los profesores y estuvo presente en gran parte de los comentarios vertidos por los alumnos.

\section{AGRADECIMIENTOS}

Héctor Cornide Reyes es beneficiario de la Beca INF-PUCV 2016 en la realización de sus estudios de Doctorado en Ingeniería Informática en la Pontificia Universidad Católica de Valparaíso.

\section{REFERENCIAS}

Anderson, L.W., D.R. Krathwohl y otros cinco autores, A taxonomy for learning, teaching, and assessing: A revision of Bloom's taxonomy of educational objectives, abridged edition, White Plains, NY: Longman (2001)

Arbizy, F. y C. Lobato, El proceso enseñanza-aprendizaje en la Universidad del País Vasco: demandas y necesidades docentes desde la perspectiva del alumnado, Revista de Psicodidáctica, 15-16, 171-188 (2003)

Bennett, B.E., D. Spencer y otros cinco autores, The flipped class manifest. The Daily Riff. Retrieved January, 15, 2015 (2012)

Bergmann, J. y A. Sams, Flip Your Classroom: Reach Every Student in Every Class Every Day, International Society for Technology in Education (2012)

Berrett, D., How 'flipping' the classroom can improve the traditional lecture, The chronicle of higher education, 12(19), 1-3 (2012)

Brame, C., Flipping the classroom, Vanderbilt University Center for Teaching (2013)

Chen, Y., Y. Wang y N.S. Chen, Is FLIP enough? Or should we use the FLIPPED model instead? Computers \& Education, 79, 16-27 (2014)

Deslauriers, L., E. Schelew y C. Wieman, Improved learning in a large-enrollment physics class, Science, 332(6031), 862864 (2011)

Estrada, M., D. Monferrer y M.A. Moliner, El Aprendizaje Cooperativo y las Habilidades Socio-Emocionales: Una Experiencia Docente en la Asignatura Técnicas de Ventas, doi:10.4067/S071850062016000600005, Formación Universitaria, 9(6), 43-62 (2016)

Foertsch, J., G. Moses, J. Strikwerda y M. Litzkow, Reversing the lecture/homework paradigm using eTEACH® webbased streaming video software, Journal of Engineering Education, 91(3), 267-274 (2002)

Griffiths, L., R. Villarroel y D. Ibacache, Implementación del modelo de aula Invertida para el aprendizaje activo de la programación en ingeniería. In XXIX Congreso Chileno de Educación en Ingeniería SOCHEDI (2016)

Hernández, M.R., V. Rodríguez, F. Parra y P. Velázquez, Las Tecnologías de la Información y la Comunicación (TICs) en la Enseñanza-Aprendizaje de la Química Orgánica a través de Imágenes, Juegos y Videos, doi: 10.4067/S071850062014000100005, Formación Universitaria, 7(1), 31-40 (2014) 
Johnson, L. y J. Renner, Effect of the flipped classroom model on secondary computer applications course: student and teacher perceptions, questions and student achievement, unpublished doctoral dissertation, University of Louisville, Louisville, Kentucky (2012)

Lage, M. J., G. J. Platt y M. Treglia, Inverting the classroom: A gateway to creating an inclusive learning environment, The Journal of Economic Education, 31(1), 30-43 (2000)

López, F.J.M., D.M. Orozco y L.G.G. Torres, Una estrategia didáctica para la enseñanza de la ingeniería de software en educación superior tecnológica, ANFEI Digital, (2), (2016)

López Rodríguez, D., M.C. García Cabanes y otros tres autores, Elaboración de material para la realización de experiencias de clase inversa (flipped classroom) (2016)

Moravec, M., A. Williams, N. Aguilar-Roca y D.K. O'Dowd, Learn before lecture: a strategy that improves learning outcomes in a large introductory biology class. CBE-Life Sciences Education, 9(4), 473-481 (2010)

Núñez, J.C., G. Vallejo y otros tres autores, Variables del estudiante, del profesor y del contexto en la predicción del rendimiento académico en Biología: análisis desde una perspectiva multinivel, Rev. de Psicodidáctica, 19(1), 145-172 (2014)

Prieto Espinosa, A., B. Prieto Campos y B. del Pino Prieto, Una experiencia de flipped classroom, XXII Jornadas sobre la Enseñanza Universitaria de la Informática, 237-244, Almería, 6-8 de julio (2016)

Rivero-Guerra, A.O., Práctica de Laboratorio de Granos de Almidón en un Curso de Universitario de Botánica General: una Experiencia de Clase Invertida, Formación Universitaria, 11(1), 87-104 (2018)

Sáez, B. y M.P. Ros, Una experiencia de flipped classroom. XI Jornadas Internacionales de Innovación Universitaria: Educar para Transformar, Villaviciosa de Odón, Madrid, 345-352 (2014)

Shim, C. Y., M. Choi y J. Y. Kim, Promoting collaborative learning in software engineering by adapting the pbl strategy. World Academy of Science, Engineering and Technology, 53, 1157-1160 (2009)

Talbert, R., Learning MATLAB in the inverted classroom. In American Society for Engineering Education, American Society for Engineering Education (2012)

Tourón, J. y R. Santiago, El modelo Flipped Learning y el desarrollo del talento en la escuela Flilpped Learning model and the development of talent at school, Revista de Educación, 368, 196-231 (2015)

Villarroel, R., H. Cornide-Reyes, R. Muñoz y T. Barcelos, Flipped classroom+ plickers, an experience to propitiate collaborative learning in software engineering. In 2017 36th International Conference of the Chilean Computer Science Society (SCCC). IEEE (2017) 
